Europhysics Letters

PREPRINT

\title{
Dynamical transitions in incommensurate systems
}

\author{
L. Consoli $\left({ }^{*}\right)$, H. Knops and A. Fasolino \\ Institute for Theoretical Physics - University of Nijmegen \\ Toernooiveld 1 - 6525 ED Nijmegen \\ The Netherlands
}

PACS. 05.45.-a - Nonlinear dynamics and nonlinear dynamical systems.

PACS. 64.60.Ht - Dynamical critical phenomena.

PACS. 68.35.-p - Solid surfaces and solid-solid interfaces: structure and energetics.

\begin{abstract}
In the dynamics of the undamped Frenkel-Kontorova model with kinetic terms, we find a transition between two regimes, a floating incommensurate and a pinned incommensurate phase. This behavior is compared to the static version of the model. While in the static case the two regimes are separated by a single transition (the Aubry transition), in the dynamical case the transition is characterized by a critical region, in which different phenomena take place at different times. In this paper, the generalized angular momentum we have previously introduced, and the dynamical modulation function are used to begin a characterization of this critical region. We further elucidate the relation between these two quantities, and present preliminary results about the order of the dynamical transition.
\end{abstract}

Introduction. - The Frenkel-Kontorova (FK) model [1] describes the interaction of a harmonic chain of atoms with a rigid substrate with period incommensurate to the lattice parameter of the chain. Its generality makes it a powerful model to investigate many different physical systems [2, 3], and in particular microscopic friction between contacting surfaces 细 6]. The static version of the model is characterized by the Aubry transition [7], from a floating to a pinned state, for a critical value $\lambda_{c}$ of the substrate modulation potential. Using the undamped dynamical version of this model, we addressed in previous papers the topic of "dissipation" (in the sense of transfer of energy from the center of mass to phonon modes) in incommensurate structures: we have studied the mechanism (parametric resonances) that governs the onset of sliding friction [6], and the conditions under which a new conserved quantity can be defined, which can be seen as a Generalized Angular Momentum (GAM) in the complex plane [8].

In this work, we present new results, showing that, in the dynamics, a floating-to-pinned transition, analogous to the static Aubry transition, is found for all values of the potential $\lambda<\lambda_{c}$. The transition is characterized by a region of critical times, with a remarkably complex behavior. After describing the FK model, we show that the dynamical modulation function undergoes at a critical time $t_{c 1}$ the same breaking of analiticity that, in the static case, occurs at $\lambda_{c}$. A second critical time $t_{c 2}>t_{c 1}$, at which the GAM conservation stops,

$\left(^{*}\right)$ E-mail: consoli@sci.kun.nl

(c) EDP Sciences 

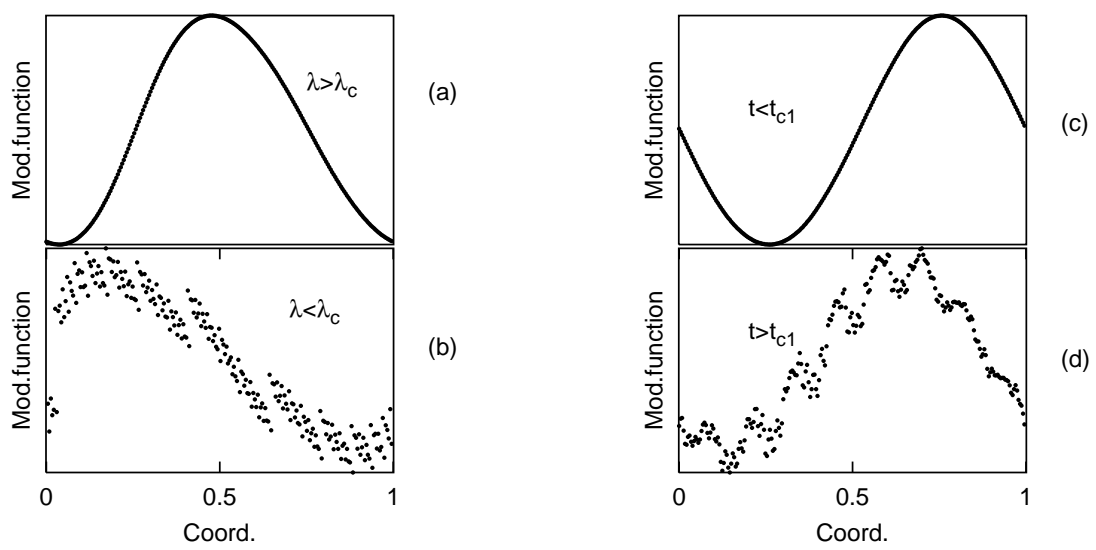

Fig. 1 - Left: the modulation function in the static FK model. (a): for $\lambda<\lambda_{c}$ the function is analytic; (b) for $\lambda>\lambda_{c}$ it becomes non-analytic. Right: two snapshots of the dynamical modulation function. (c) $N=233, \lambda=0.05, t=300<t_{c 1}$; (d) $N=233, \lambda=0.05, t=820>t_{c 1}$. Even if $\lambda<\lambda_{c}$, the modulation function becomes non-analytic.

was identified in [8]; here, we show explicitly the connection between these two quantities, by showing analytically that the analiticity of the modulation function implies conservation of the GAM. We also present some initial results about the order of this dynamical transition.

The static model. - The FK model describes a chain of $N$ atoms in contact with a substrate, described by a rigid periodic potential. The intrachain interaction is modelled by a first neighbor harmonic potential, and the total potential energy reads:

$$
\mathcal{V}=\sum_{n}\left[\frac{1}{2}\left(u_{n+1}-u_{n}-l\right)^{2}+\frac{\lambda}{2 \pi} \sin \left(\frac{2 \pi u_{n}}{m}\right)\right]
$$

where $u_{n}$ are the lattice positions, $l$ is the average atomic distance, and $\lambda$ is the strength of the substrate potential rescaled to the spring constant. The ratio between $l$ and the modulation potential period $m$ is chosen to be an irrational number (in our case, $m=1$ and $l=\tau=(\sqrt{5}+1) / 2$, the golden mean), in order to simulate an incommensurate (IC) modulated structure. Numerically, we use a finite chain of length $\mathrm{Nl}$ and use ratio of consecutive Fibonacci numbers to approximate incommensurability [6]. The Aubry transition can be monitored by the modulation function (see e.g. [3]). It is defined as: $f(n l \bmod m)=u_{n}-n l-Q$, where $Q=\frac{1}{N} \sum_{n} u_{n}$ is the center of mass $(\mathrm{CM})$ of the chain, and contains information about the groundstate positions of the chain particles with respect to the substrate. When $\lambda>\lambda_{c}$, it becomes non-analytic, as shown in the left side of fig. 11. Another feature of the FK model is the existence of a zero frequency phason mode below $\lambda_{c}$ that moves to finite frequency at the Aubry transition. If the interaction with the substrate is weak $\left(\lambda<<\lambda_{c}\right)$, deviations from equidistant spacing $l$ in the groundstate are modulated with the substrate modulation wave-vector $q=2 \pi l$, due to the frozen-in phonon $\omega_{q}$. For values of the coupling $\lambda<\lambda_{c}$, the Fourier transform of the displacement related to $q$ has thus the strongest amplitude, and amplitudes of higher harmonics $n q$ scale with $\lambda^{|n|}$. It is therefore natural to use $|n|$ instead of $k$ and relabel the modes (see [8] for the details of the relabeling procedure). In the left side of fig. 2, it can be seen that, both with the usual labels (panel (a)) and with the relabeling (panel (b)), the effect of the modulation is lost when $\lambda>\lambda_{c}$, which for this model is $\lambda_{c}=0.154 \ldots$. 
The dynamical model. - In the dynamical version of the model, a kinetic term is added to the potential energy; in our case, it corresponds to giving an initial velocity to the particles of the chain. The Hamiltonian now reads:

$$
\mathcal{H}=\mathcal{V}+\mathcal{K}=\mathcal{V}+\sum_{n} \frac{p_{n}^{2}}{2}
$$

where the $p_{n}$ are the particle momenta. The CM velocity is defined as: $P=\frac{1}{N} \sum_{n} p_{n}$. The dynamics of the system can be studied by following the time evolution of the CM of the chain $\mathrm{Q}$, and of the particles deviations $x_{n}=u_{n}-n l-Q$. As initial conditions we choose $p_{n}(t=0)=P_{0}$ and $x_{n}(t=0)$ corresponding to the groundstate. The equations of motion for the deviations are numerically solved with a Runge-Kutta-Fehlberg algorithm. Convergence of the results is found for a tolerance of $10^{-9}$, ensuring total energy conservation up to $10^{-8}$. As we saw in the previous section, phonon amplitudes play a crucial role in the statics. In the dynamics, their behavior can be used to elucidate the time evolution of the system. The inherent nonlinear coupling of the CM motion to the phonons of the chain leads to an irreversible decay of the CM velocity, that in an Hamiltonian picture can be identified with the onset of sliding friction. Transfer of kinetic energy from the CM to the internal vibrations occurs via a complex sequence of parametric resonances mediated by ordinary resonances with phonons related to the modulation potential [6]. These excitations can further combine and give rise to the appearance of Umklapp terms. This phenomenon can be monitored by the breakdown of the conservation of the GAM, as derived in [8]:

$$
p_{\phi}=-i \sum_{n} n q a_{-n q} \dot{a}_{n q}+\frac{q}{2 \pi} \dot{Q} \equiv L+\frac{q}{2 \pi} \dot{Q}
$$

where $a_{ \pm n q}$ are the phonon amplitudes, obtained by Fourier transforming at each timestep the expression for the chain distorsions $x_{n}$.

Modulation function and phonon amplitudes. - We have shown in the previous section how the behavior of the modulation function changes when going through the transition at $\lambda_{c}$. If we now turn to the dynamical FK model with initial velocity, it is very intriguing to see that, for all values $\lambda<\lambda_{c}$, the same behavior is found, after a critical time $t_{c 1}$.

This can be shown by extending the concept of modulation function to the dynamics. Since the value $q=2 \pi l$ is fixed, we do it in a straightforward way: $f_{t}(n l \bmod 1) \equiv u_{n}(t)-$ $n l-Q(t) \equiv x_{n}(t)$. The results are shown in panels (c) and (d) of fig. 11. The similarity with the static case is apparent. The time $t_{c 1}$ can be accurately determined from the largest discontinuity of the modulation function (see fig. 3, lower panels). Another signature of the dynamical transition is that it is accompanied by a breakdown of the conservation of the GAM. This is shown in the middle panels of fig. 3. It is however remarkable that this breakdown always occurs at a later time $t_{c 2}>t_{c 1}$. Fig. A shows that this remains true for all system sizes considered. Qualitatively, one can understand this because the non-analiticity of the modulation function is caused by the excitation of so many phonon modes as to render the Fourier series representing it not absolutely convergent. The conservation of the GAM only breaks down when this excitation reaches the zone boundary, generating Umklapp terms [8]. This is shown more formally in the next section. This dynamical transition can also be seen in the qualitative difference in the Fourier amplitudes shown in panels (c) and (d) of fig. 2 .

The GAM and the modulation function. - We show that analiticity of the modulation function implies conservation of the GAM. The modulation function can be written as:

$$
f(z)=\sum_{m} a_{m q} e^{i 2 \pi m z}
$$



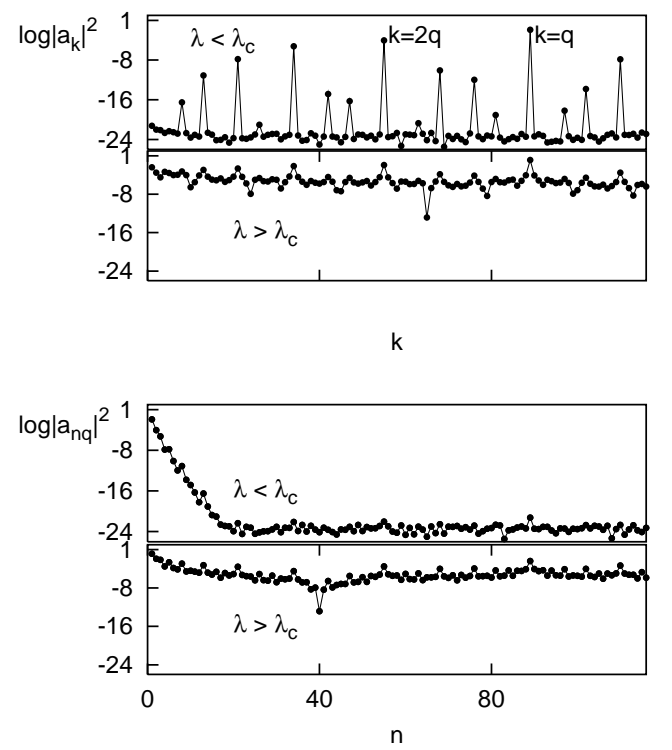

(a)

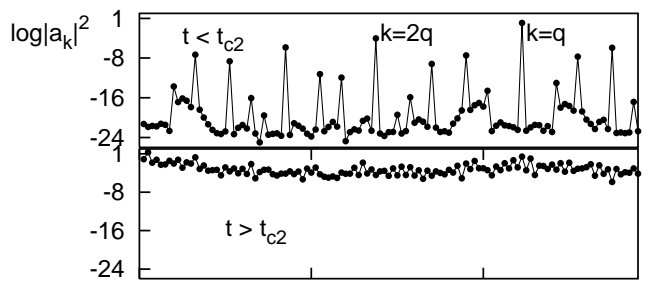

$\mathrm{k}$

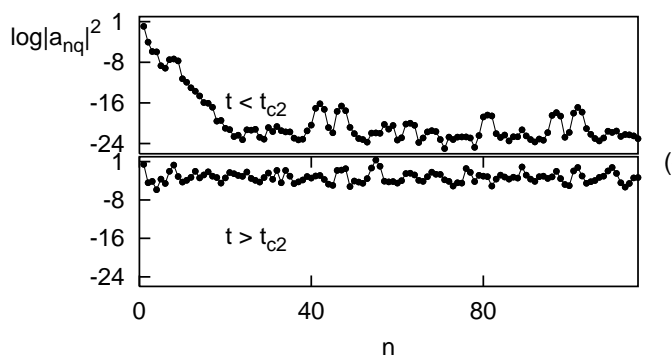

Fig. 2 - (a) Phonon amplitudes in the static FK model, for the cases $N=233, \lambda=0.05<\lambda_{c}$, and $\lambda=0.16>\lambda_{c}$. The modes are labeled in the usual way. The first two modulation wave-vectors $k=q, 2 q$ are shown. (b) Same, but the relabeling $k \rightarrow n$ is used to make the scaling behavior apparent. (c) Phonon amplitudes in the dynamical case, for $N=233, P_{0}=0.29, \lambda=0.05<\lambda_{c}$, $t=300<t_{c}$, and $t=1000>t_{c}$. Modes are labeled as in (a). (d) Same, with relabeling as in (b).
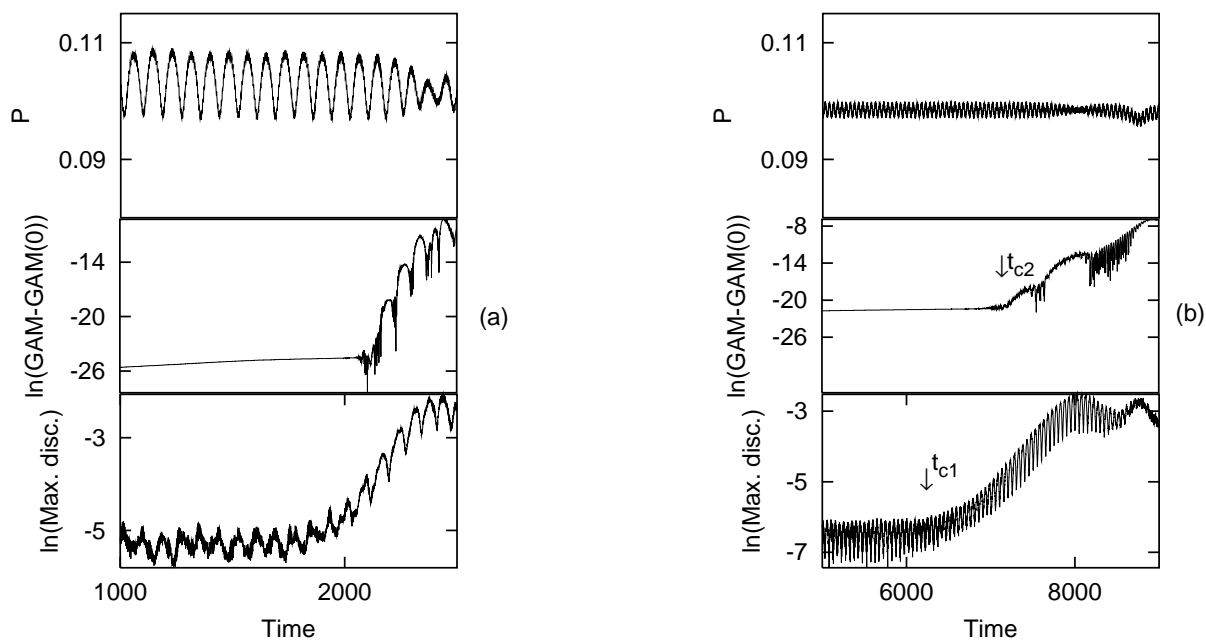

Fig. 3 - Behavior of the deviations of the GAM from the initial value $\operatorname{GAM}(0)$ and of the largest discontinuity of the modulation function. (a) $N=144, \lambda=0.05, P_{0}=0.11$. This velocity corresponds to the resonance $2 \pi P_{0}=\omega_{2 q} / 2$. (b) Same $N$ and $\lambda$, but with $P_{0}=0.1$. The two values of $P_{0}$ are very close, but the behavior of the $\mathrm{CM}$ momentum is quite different since the second value is not a resonance. Nevertheless, the GAM and the largest discontinuity have the same kind of temporal evolution. This stresses the generality of the transition mechanism. 


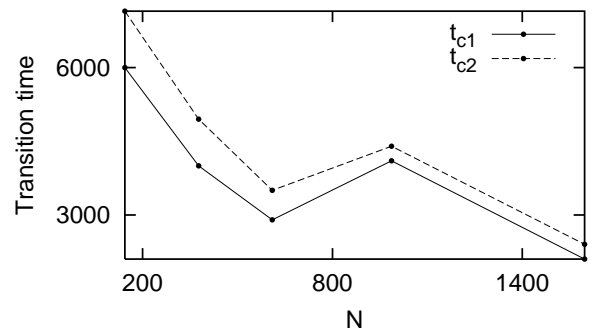

Fig. 4 - Behavior of $t_{c 1}$ and $t_{c 2}$ as a function of the number of particles $N$, for $P_{0}=0.1, \lambda=0.05$, and $N=144,377,610,987,1597$. For all cases, $t_{c 2}>t_{c 1}$.

Indeed, writing the distortions $x_{n}$ as inverse Fourier transforms, with mode relabeling, gives:

$$
x_{n}=\sum_{m} a_{m q} e^{i n m q}
$$

From eqs. (4) and (5) it follows immediately that:

$$
x_{n}=f(n l)
$$

This is true for the statics; taking into account the time dependence, we can generalize to the dynamics: $a_{m} \rightarrow a_{m}(t), f(z) \rightarrow f(z, t)$. The equations of motion for the distorsions are $\left(^{1}\right)$ :

$$
\ddot{x}_{n}+\ddot{Q}=x_{n+1}+x_{n-1}-2 x_{n}+\lambda \cos \left(n q+2 \pi x_{n}+2 \pi Q\right)
$$

From eqs. (6) and (7), we derive the equation of motion for $f$ :

$$
\ddot{f}=f(z+l, t)+f(z-l, t)-2 f(z, t)-\ddot{Q}+\lambda \cos (2 \pi z+2 \pi f(z, t)+2 \pi Q(t))
$$

where we make the association $x_{n \pm 1}=z \pm l$. Let us now take the first of the two terms that make up the GAM in eq. (3) and rewrite it in terms of the modulation function. If the modulation function $f$ is analytic, we can express the coefficients $a_{n q}$ as: $a_{n q}=\int_{0}^{1} d z e^{-i n 2 \pi z} f(z, t)$ and using partial integration, we obtain:

$$
L=-i \sum_{n} n q a_{-n q} \dot{a}_{n q}=\frac{q}{2 \pi} \int_{0}^{1} d z \frac{d f(z, t)}{d z} \dot{f}(z, t)
$$

We now compute the first time derivative of eq. (9) It can be shown that, due to periodic integration boundaries, only one term remains:

$$
\dot{L}=\frac{q}{2 \pi} \lambda \int_{0}^{1} d z \frac{d f(z, t)}{d z} \cos (2 \pi(z+f(z, t)+Q(t))
$$

Using $f+z$ as a new integration variable and periodic integration boundaries, we get:

$$
\dot{L}=-\frac{q}{2 \pi} \lambda \int_{0}^{1} d z \cos (2 \pi(f(z, t)+z+Q(t))
$$

$\left({ }^{1}\right)$ In eq. (2) of ref. [6] and eq. (4) of ref. [8] the $\ddot{Q}$ term has been omitted. 
From eq. (7), we can now obtain the expression for the equation of motion of the CM $Q$ :

$$
\ddot{Q}=\frac{\lambda}{N} \sum_{n} \cos \left(n q+2 \pi x_{n}+2 \pi Q\right)
$$

Under the assumption that we made of the analiticity of $f$, we can go from the summation to the integral and, using eq. (8), we obtain:

$$
\ddot{Q}=\lambda \int d \phi \cos (2 \pi(z+f(z, t)+Q(t))
$$

Comparison between eqs. (11) and (13) gives us (after integration):

$$
L+\frac{q}{2 \pi} \dot{Q}=\text { const }
$$

i.e., we have derived the conservation of the GAM from the definition of the modulation function.

The crucial assumption in the calculation is the analiticity of the modulation function, which in the static case defines the regime under the Aubry transition. Notice that this result is compatible with our observation that $t_{c 2}>t_{c 1}$ : analiticity of the modulation function implies GAM conservation, but the contrary is not necessary. Lastly, we notice from fig. 3 that this mechanism is robust: while resonances affect in a significant way the CM velocity, the GAM and the modulation function retain the same kind of behavior.

Order of the transitions and relation with the static case. - Both transitions we observe in the dynamical case are very sharp; this suggests that, in the limit of an infinite chain, they could be first-order. This would be in contrast with the static case, where it has been proven (see e.g. (3)) that the largest discontinuity in the modulation functions undergoes a second order transition. Presently, we do not have the analytical tools to check this rigorously. We can however get some numerical indications: we fit the behavior of the GAM and of the largest discontinuity to an exponential function, and study the behavior of the exponent as a function of the size $N$. In fig. 5 , we see that, in both cases, the value of the fitting exponent grows when $N$ increases. This indicates that, for $N \rightarrow \infty$, the exponent could diverge and the transition would become first-order. More work is needed to confirm these initial results. It is also possible to relate in a direct way the dynamical and static behavior. This is shown in fig. 6 for $t_{c 2}$ : when the substrate potential approaches the critical static value $\lambda_{c}, t_{c 2}$ goes to zero. This allows us to relate the statics and the dynamics of the FK model.

Conclusions. - We presented in this paper numerical and analytical evidence that, in the kinetic FK model, a region is found where dynamical transitions take place. This region separates a floating IC from a pinned IC phase, and is therefore equivalent to the static Aubry transition (and, in fact, reducing to it in the limit of $\lambda \rightarrow \lambda_{c}$ ). Non-analiticity of the modulation function and breakdown of conservation of the GAM characterize these transitions. More work is needed to assess the order of these transitions and the relationship between $t_{c 1}$ and $t_{c 2}$, investigating for example the possibility that, for $N \rightarrow \infty, t_{c 1}$ and $t_{c 2}$ converge to a single value $t_{c}$.

We would like to thank Ted Janssen for productive discussions. 

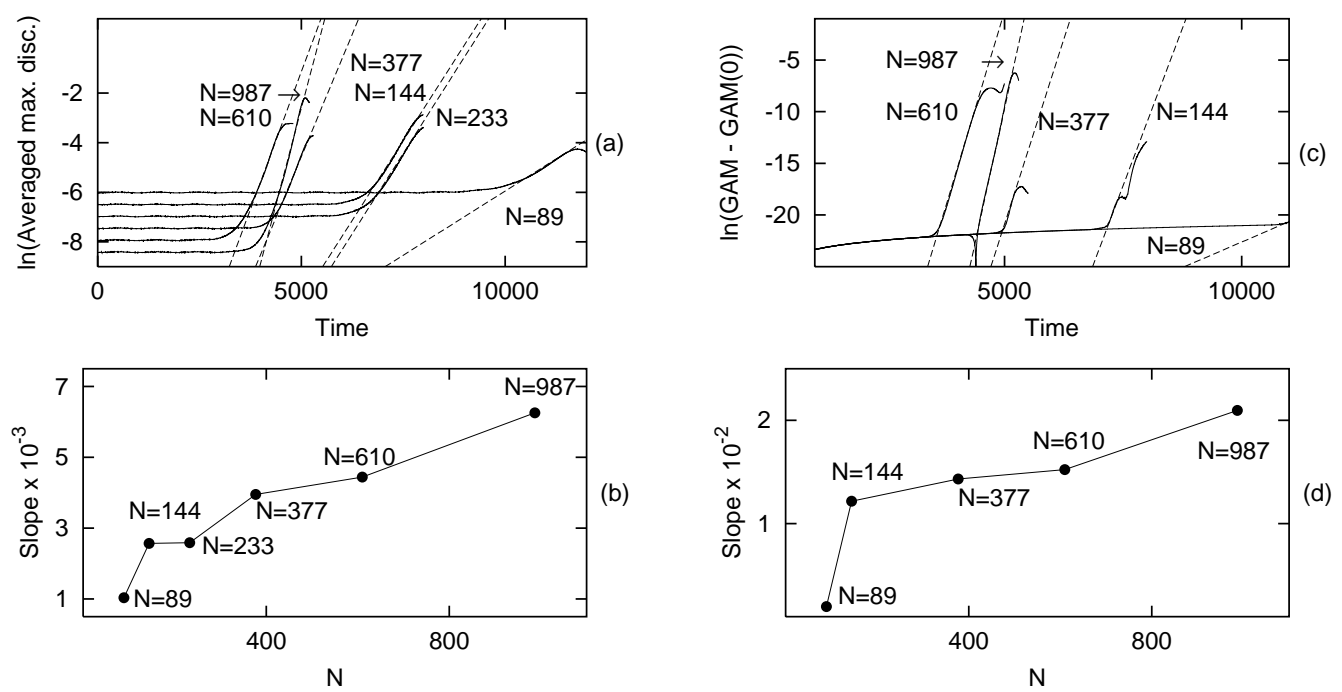

Fig. 5 - (a) Exponential fits for the growth of the largest discontinuity in the modulation function for various $N$ at $\lambda=0.05, P_{0}=0.10$. The initial values are determines by numerical accuracy. (b) Behavior of the fitting exponents shown in (a); the increase with $N$ is apparent. (c) Exponential fits for the GAM. (d) Exponents of the GAM fit as function of $N$; here too, they increase with $N$.

\section{REFERENCES}

[1] Frenkel, Ya. I. and Kontorova, T. A., Zh. Eksp. Teor. Fiz., 8 (1938) 89

[2] Aubry S., J. Phys (France, 44 (1983) 147

[3] Floría L. M. and Mazo J. J., Adv. Phys., 45 (1996) 505

[4] Strunz T. and Elmer F. J., Phys. Rev. E, 58 (1998) 1601

[5] Braun O. M., Hu B. and Zeltser A., Phys. Rev. E, 62 (2000) 4235

[6] Consoli L., Knops H. and Fasolino A., Phys. Rev. Lett., 85 (2000) 302

[7] Peyrard M. and Aubry S., J. Phys. C, 16 (1983) 1953

[8] Consoli L., Knops H. and Fasolino A., Phys. Rev. E, 64 (2001) 016601

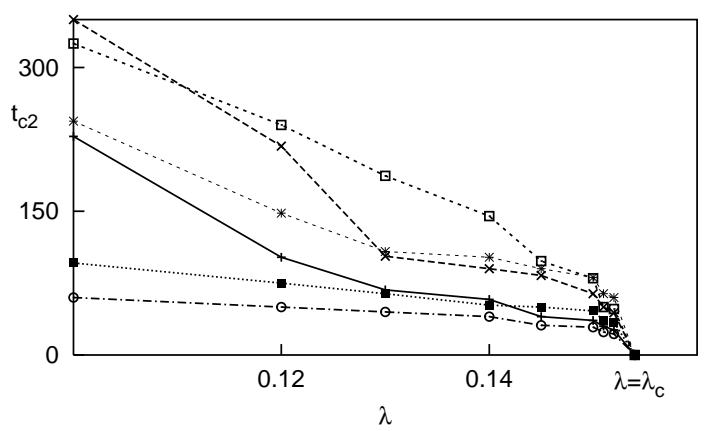

Fig. 6 - Behavior of the transition time $t_{c 2}$ as a function of the substrate potential $\lambda$ in the proximity of $\lambda_{c}=0.154 \ldots$ Each line corresponds to a different initial velocity. $t_{c 2} \rightarrow 0$ for $\lambda \rightarrow \lambda_{c}$. 